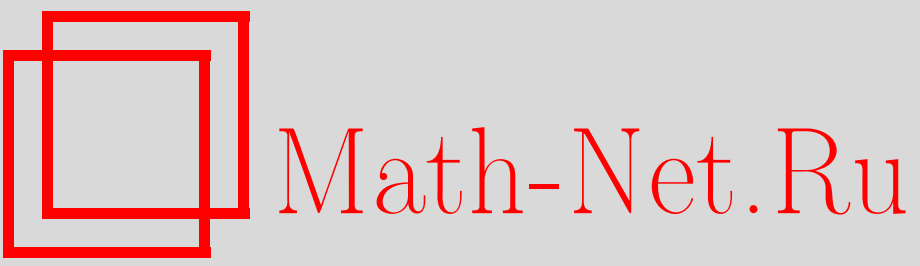

А. А. Туганбаев, Идеалы наследственных колец, УМН, 2003, том 58, выпуск 2, 177-178

DOI: https://doi.org/10.4213/rm624

Использование Общероссийского математического портала Math-Net.Ru подразумевает, что вы прочитали и согласны с пользовательским соглашением

http://www.mathnet.ru/rus/agreement

Параметры загрузки:

IP: 54.166 .219 .16

26 апреля 2023 г., 18:34:35 


\section{ИДЕАЛЫ НАСЛЕДСТВЕННЫХ КОЛЕЦ}

\section{А. А. ТУГАНБАЕВ}

Колшц называется инвариантнылм справа (квазиинвариантным справа), если каждый его правьй идеал (максимальньй правьй идеал) является идеалом.

Теорема 1. Пусть А-наследственное справа кольцо.

(1) $A$ - нётерово справа кольцо тогда и только тогда, когда каждый замкнутый правый идеал кольца $A$ конечно порожден.

(2) $A$ - инвариантное справа кольцо тогда и только тогда, когда для каждого әлемента а кольца $A$ существует такое $n=n(a)$, что $a^{n} A-$ идеал кольца $A$.

Кольцо $A$ назьвается локализуемым справа, если для любого его максимального правого идеала $M$ существуют такие кольцо $A_{M}$ и колцевой гомоморфизм $f_{M}=f: A \rightarrow A_{M}$, что все элементы множества $f(A \backslash M)$ обратимы в $A_{M}, A_{M}=\left\{f(a) f(s)^{-1} \mid a \in A, s \in A \backslash M\right\}$ и $\operatorname{Ker}(f)=\{a \in A \mid$ as $=0$ для некоторого $s \in A \backslash M\}$. В этом случае $A_{M}$ называется правой локализачией кольца $A$ относительно $M$, а $f_{M}$ - каноническим гомоморфизмом.

Лемма 1. Пусть каждый замкнутый подмодуль модуля $M_{A}$ конечно порожден.

(1) Если каждый подмодуль модуля $M$ является прямой суммой конечно порожденных модулей, то $M$ - нётеров модуль.

(2) Если $M$ - наследственный модуль и $A$ - полунаследственное справа кольцо, то М - нётеров модуль.

ДокАЗАТЕЛЬСтво. (1) Допустим противное. Пусть $\Lambda$ - непустое множество всех подмодулей модуля $M$, не являющихся конечно порожденными. Тогда $\Lambda$ содержит объединение любой цепи своих элементов. По лемме Цорна $\Lambda$ содержит максимальный элемент $N$. По условию $N=$ $\bigoplus_{i=1}^{\infty} N_{i}$, где все $N_{i}$ - ненулевые конечно порожденные модули. Тогда $N=X \oplus Y$, где $X$-прямая сумма всех $N_{i}$ с нечетными индексами и $Y$ - прямая сумма всех $N_{i}$ с четными индексами. $X$ и $Y$ не являются конечно порожденными. По лемме Цорна существует такой замкнутьй подмодуль $\bar{X}$ модуля $M$, что $\bar{X}$ - существенное расширение модуля $X$. Тогда $\bar{X} \cap Y=0$. По условию $\bar{X}$ конечно порожден. Поэтому $X \neq \bar{X}$ и $\bar{X} \oplus Y$ строго содержит максимальньй элемент $X \oplus Y$ из $\Lambda$. Тогда $\bar{X} \oplus Y$ конечно порожден и $Y$ конечно порожден. Получено противоречие.

(2) следует из (1) и того, что каждый проективньй правый модуль над полунаследственным справа колцом является прямой суммой конечно порожденных модулей $[1 ; 39.13(2)]$.

ЛЕмма 2. Пусть $A$ - локализуемое справа кольцо.

(1) Если для любого максимального правого идеала $М$ кольца А правая локализация $A_{M}$ является инвариантным справа кольцом, то $A$ - инвариантное справа кольцо.

(2) Если для любого максимального правого идеала $M$ кольца $A$ правая локализация $A_{M}$ является цепным справа кольцом, то $A$ - дистрибутивное справа кольцо.

(3) Если $A$ - риккартово справа кольцо, то для любого максимального правого идеала $M$ кольца $A$ правая локализачия $A_{M}$ является локальной областью.

(4) Если $A$ - наследственное справа кольцо, то для любого максимального правого идеала $M A_{M}$ наследствена справа.

(5) Если $M$ - максимальньй правьй идеал кольца $A$ и $f_{M}: A \rightarrow A_{M}-$ канонический гомоморфизм, причем $A / \operatorname{Ker}\left(f_{M}\right)$ - равномерное справа кольцо, то правая локализачия $A_{M}$ является равномерныц справа кольцом.

Лемма 2 следует из утверждений 5.26(6), 6.23(4), 6.25(1), 6.22(14) и 5.23(11) в [2].

Лемма 3. Пусть $A$ - наследственное справа локализуемое справа кольцо.

(1) Для любого его максимального правого идеала $M A_{M}$ является наследственной справа локальной областью, у которой все правые идеаль свободнь.

(2) Если для любого максимального правого идеала $M$ кольца $A A_{M}$ является равномерным справа кольцом, то $A$ - инвариантное справа дистрибутивное справа кольцо

Работа выполнена при поддержке Российского фонда фундаментальных исследований (грант № 99-01-00382). 
и для любого максимального правого идеала $M A_{M}$ является инвариантной справа цепной справа областью главньх правых идеалов.

ДокАЗАТЕЛЬСтво. По леммам 2(3) и 2(4) $A_{M}$ - наследственная справа локальная область. Каждьй правый идеал кольца $A_{M}$ является проективньм модулем над локальным кольцом $A_{M}$ и поэтому свободен.

(2) Пусть $M$ - максималшный правый идеал. Так как каждый равномерньй свободньй модуль цикличен, то из (1) следует, что равномерное справа кольцо $A_{M}$ является локальной областью главных правых идеалов. Поэтому $A_{M}$ - инвариантная справа цепная справа область. По леммам $2(1)$ и $2(2) A$ - инвариантное справа дистрибутивное справа кольцо.

Лемма 4. Пусть $A$ - кольцо и для каждого әлемента а кольца $A$ существует такое натуральное число $n=n(a)$, что $a^{n} A-$ идеал кольца $A$.

(1) $A$ - квазичнвариантное справа нормальное кольцо и для любого максимального правого идеала $M$ кольца $A$ подмножество $A \backslash M$ перестановочно справа.

(2) Для любого вполне первичного идеала $N$ кольца $A$ область $A$ равномерна справа.

(3) Если $A$ - риккартово справа кольцо, то $A$ - локализуемое справа кольцо и для любого его максимального правого идеала $M$ правая локализация $A_{M}$ является равномерной справа локальной областью.

(4) Если $A$ наследственно справа, то $A$ - инвариантное справа дистрибутивное справа кольщо и для любого максимального правого идеала $M$ правая локализачия $A_{M}$ является инвариантной справа цепной справа областью главных правых идеалов.

ДокаЗАТЕльство. (1) Если $e=e^{2} \in A$, то $e^{n}=e$ и $(1-e)^{n}=1-e$ для всех $n \geqslant 1$. Тогда из условия следует, что $e A$ и $(1-e) A$ - идеалы. Поэтому $A$ нормально. Пусть $M-$ максимальный правый идеал. Докажем, что $M$ - идеал. Пусть $B$ - наибольший идеал, лежащий в $M$. Допустим, что $B \neq M$. Так как $A / B$ полупримитивно, то не все элементы ненулевого правого идеала $M / B$ нильпотентны. Поэтому существует $m \in M$, что $m^{n} \notin B$ для всех $n$. По условию существует такое натуральное число $n$, что $m^{n} A$ - идеал. Тогда $B+m^{n} A$ - такой идеал, что $B \subsetneq B+$ $m^{n} A \subseteq M$. Получено противоречие. Поэтому $A$ квазиинвариантно справа. Пусть $S=A \backslash M$. Докажем, что $S$ перестановочно справа. Пусть $a \in A$ и $s \in S$. По условию существует такое $k$, что $a s^{k}=s^{k} b$ для некоторого $b \in A$. Так как $M$ - максимальный правый идеал, являющийся идеалом, то $A / M$ - тело. Поэтому $s^{k} \in S, a s^{k}=s s^{k-1} b \in a S \cap s A$ и $T$ перестановочно справа.

(2) Пусть $\bar{a}$ и $\bar{b}$ - ненулевые элементы области $A / N$ и $h: A \rightarrow A / N$ - естественньй эпиморфизм. Существуют такие $a, b \in A \backslash N$, что $h(a)=\bar{a}$ и $h(b)=\bar{b}$. По условию существуют такие $m$ и $n$, что $a^{m} A$ и $b^{n} A$ - идеалы. Так как $h(A)$ - область и $a, b \in A \backslash N$, то $h\left(a^{m} A\right)$ и $h\left(b^{n} A\right)$ - ненулевые идеалы области $h(A)$. Поэтому $h\left(a^{m} A\right) h\left(b^{n} A\right) \neq 0$. Тогда $h\left(a^{m} A\right) \cap$ $h\left(b^{n} A\right) \supseteq h\left(a^{m} A\right) h\left(b^{n} A\right) \neq 0$ и область $h(A)$ равномерна справа.

(3) Риккартово справа нормалшное колњц $A$ редуцированно $[2 ; 5.28]$. По $[2 ; 5.19(4)] A$ локализуемо справа. Пусть $M$ - максимальньй правый идеал и $f: A \rightarrow A_{M}$-канонический кольцевой гомоморфизм. По лемме $2(3) A_{M}$ - локальная область. Так как $A_{M}$ - область, то $A / \operatorname{Ker}(f)-$ область. По (2) $A / \operatorname{Ker}(f)$ равномерна справа. По лемме $2(5)$ область $A_{M}$ равномерна справа.

(4) По (3) $A$ локализуемое справа и для любого максимального правого идеала $M A_{M}$ является равномерной справа локальной областью. По лемме $3(2) A$ - инвариантное справа дистрибутивное справа колшцо и для любого максимального правого идеала $M$ правая локализация $A_{M}$ является инвариантной справа цепной справа областью главных правых идеалов.

ОКОНЧАнИЕ дОКАЗАТЕЛЬСТВА ТЕОРЕМЫ. Теорема следует из лемм 1(2) и 4(4).

\section{СПИСОК ЛИТЕРАТУРЫ}

[1] R. Wisbauer. Foundations of Module and Ring Theory. Philadelphia: Gordon and Breach, 1991. [2] A. A. Tuganbaev. Semidistributive Modules and Rings. Dordrecht: Kluwer, 1998.

Московский энергетический институт (технический университет)

E-mail: askar@tuganbaev.mccme.ru
Принято редколлегией 03.02 .2003 